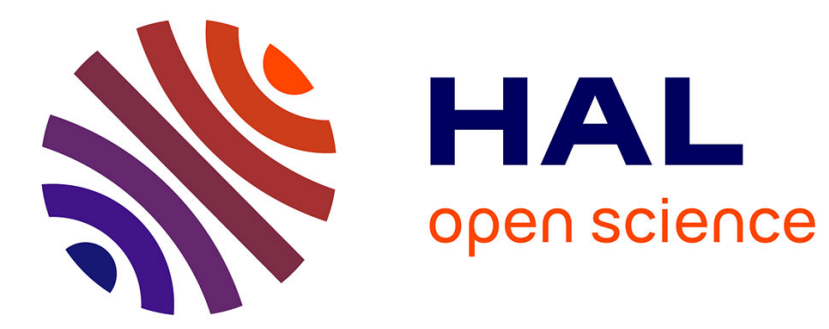

\title{
Counterexamples to Symmetry for Partially Overdetermined Elliptic Problems
}

Ilaria Fragalà, Filippo Gazzola, Jimmy Lamboley, Michel Pierre

\section{To cite this version:}

Ilaria Fragalà, Filippo Gazzola, Jimmy Lamboley, Michel Pierre. Counterexamples to Symmetry for Partially Overdetermined Elliptic Problems. Analysis, 2009, 29 (1), pp.85-93. hal-00362312

\section{HAL Id: hal-00362312 \\ https://hal.science/hal-00362312}

Submitted on 17 Feb 2009

HAL is a multi-disciplinary open access archive for the deposit and dissemination of scientific research documents, whether they are published or not. The documents may come from teaching and research institutions in France or abroad, or from public or private research centers.
L'archive ouverte pluridisciplinaire HAL, est destinée au dépôt et à la diffusion de documents scientifiques de niveau recherche, publiés ou non, émanant des établissements d'enseignement et de recherche français ou étrangers, des laboratoires publics ou privés. 


\title{
Counterexamples to Symmetry for Partially Overdetermined Elliptic Problems
}

\author{
Ilaria Fragalà, Filippo Gazzola, Jimmy Lamboley, Michel Pierre
}

July 2008

Summary: We exhibit several counterexamples showing that the famous Serrin's symmetry result for semilinear elliptic overdetermined problems may not hold for partially overdetermined problems, that is when both Dirichlet and Neumann boundary conditions are prescribed only on part of the boundary. Our counterexamples enlighten subsequent positive symmetry results obtained by the first two authors for such partially overdetermined systems and justify their assumptions as well.

\section{Introduction}

Let $\Omega$ be an open bounded connected subset of $\mathbb{R}^{n}$ with smooth enough boundary, and let $\Gamma$ be a nonempty connected (relatively) open subset of $\partial \Omega$. Let also $\nu$ denote the unit outer normal to $\partial \Omega, c$ be a positive constant and $f: \mathbb{R} \rightarrow \mathbb{R}$ be a smooth function. By "overdetermined problem", we mean any boundary value problem of the following kind:

$$
\begin{cases}-\Delta u=f(u) & \text { in } \Omega \\ u=0 \quad \text { and } \quad u_{\nu}=-c & \text { on } \Gamma \\ u=0 & \text { on } \partial \Omega \backslash \Gamma,\end{cases}
$$

or

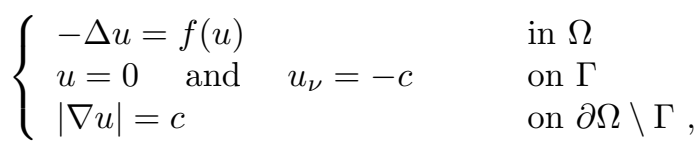

where $u_{\nu}$ denotes the normal derivative of $u$ on $\partial \Omega$. Here and in the sequel, by a solution $u$ to problem (11) (resp. (2)), we always mean that $u \in \mathcal{C}^{0}(\bar{\Omega}) \cap \mathcal{C}^{1}(\Omega \cup \Gamma) \cap \mathcal{C}^{2}(\Omega)$ (resp. $\left.\mathcal{C}^{1}(\bar{\Omega}) \cap \mathcal{C}^{2}(\Omega)\right)$.

The choice of the word "overdetermined" is justified by the presence of both the Dirichlet and Neumann conditions on a same nonempty part $\Gamma$ of the boundary in problems (11)-(2): this makes them in general not well-posed. Thus the existence of a solution to (11) or (2) is not always guaranteed, and, if existence happens to hold, it is actually supposed to imply some severe geometric constraint on $\Omega$.

This kind of problem was studied by Serrin 14. His celebrated result states that, in the case of totally overdetermined problems, that is when $\Gamma \equiv \partial \Omega$, then existence of a solution implies that $\Omega$ is a ball (and $u$ is radially symmetric).

More recently, the case of partially overdetermined problems, that is when $\Gamma \varsubsetneqq \partial \Omega$, has been studied by the first two authors in [8], where they investigate the following natural question:

"If $\Gamma \varsubsetneqq \partial \Omega$, can we still conclude that $\Omega$ is a ball

whenever (11) or (2) admits a solution?"

The answer is trivially no without any extra natural geometric restriction on $\Omega$. Assume, for instance, that $\Omega$ is an annulus, that is $\Omega=\left\{x \in \mathbb{R}^{n} ; 0<a<|x|<b\right\}$. Then, the solution of $-\Delta u=1$ on $\Omega$, with $u=0$ on its boundary, is radially symmetric. Therefore, $u_{\nu}$ is equal to a constant on each piece of the boundary, but with different constants for each of them.

AMS 1991 subject classification: Primary: 35J70; Secondary: 35B50,49Q10

Key words and phrases: overdetermined boundary value problems, shape optimization 
On the other hand, if $\partial \Omega$ is assumed to be connected, the problem becomes much more significant and delicate. In fact there are many different situations where the answer to the above question is yes, so that Serrin's symmetry result continues to hold. This occurs under suitable additional assumptions, involving both regularity and geometric features, on the source term $f$ and the overdetermined region $\Gamma$ : for the detailed statements, as well as for a more extensive bibliography about overdetermined problems, we refer to [8].

The goal of this note is to show that there are nontrivial cases (meaning in particular that $\partial \Omega$ is connected) when the requirements of [8] are not satisfied and problems like (11)-(2) admit a solution in domains $\Omega$ different from a ball.

The counterexamples we construct for problems of type (1) or (2) are of different kind. Problems of type (1) are treated in Section 2 by an approach based on shape optimization and domain derivative. More precisely, we consider the problem of minimizing the Dirichlet energy of domains with prescribed volume and confined in a planar box, that is

$$
\left|\Omega^{*}\right|=\alpha, \Omega^{*} \subset D, J\left(\Omega^{*}\right)=\min _{|\Omega|=\alpha, \Omega \subset D} J(\Omega),
$$

where $D=(-1,1)^{2}$ and

$$
J(\Omega):=\inf _{v \in H_{0}^{1}(\Omega)}\left\{\int_{\Omega}\left(\frac{1}{2}|\nabla v|^{2}-v\right) d x\right\} .
$$

Choosing $\alpha$ in a suitable range and applying the regularity results in [1, 2], we obtain that (3) admits an optimal open shape $\Omega^{*}$ with a nonempty smooth "free boundary" $\partial \Omega^{*} \cap D$. Then, writing down the optimality conditions by using shape derivatives, we are lead to a problem of type (11) on $\Omega^{*}$, with $f \equiv 1$ and $\Gamma=\partial \Omega^{*} \cap D$.

Problems of type (2) are treated in Section 3 by a different approach, which works in any dimension $n \geq 2$. In this case, the counterexamples are derived through some explicit computations. They are based on the idea of studying the zero level surfaces of radial functions $u$ built so as to satisfy both an elliptic equation of the type $-\Delta u=f(u)$ on the whole $\mathbb{R}^{n}$ and the eikonal equation $|\nabla u|=c$ on the complement of a ball. Such construction can be adapted to treat also the case of a partially overdetermined problem similar to (2), but stated on an exterior domain (see Section 3.2 .

\section{Counterexamples using shape optimization}

In this section we use shape optimization in order to prove the following.

Theorem 2.1 There exists an open starshaped planar domain $\Omega \subset(-1,1)^{2}$, different from a disk, such that, for a nonempty connected analytic subset $\Gamma$ of $\partial \Omega$, the problem

$$
\left\{\begin{array}{ccccc}
-\Delta u & = & \text { in } & \Omega \\
u & = & 0 & \text { on } & \partial \Omega \\
u_{\nu} & = & -c & \text { on } & \Gamma
\end{array}\right.
$$

admits a solution.

Remark 2.2 Note that a nonempty analytic subset $\Gamma$ of $\partial \Omega$ is relatively open in $\partial \Omega$.

The interest of this negative result should be considered in the light of the following extension of Serrin's result proved in [8]:

Proposition 2.3 Let $\Omega$ be open and bounded with $\partial \Omega$ connected. Let $\Gamma \subset \partial \Omega$ nonempty and (relatively) open. Assume there exists an open set $\widetilde{\Omega}$ with a connected analytic boundary containing $\Gamma$. If there exists a solution $u$ of (1) with $f$ analytic, then $\Omega=\widetilde{\Omega}, \Omega$ is a ball, and $u$ is radially symmetric. 
In particular, Proposition 2.3 implies that the analytic piece $\Gamma$ of the boundary of $\Omega$ found in Theorem 2.1 cannot be continued into a globally analytic closed "curve" (namely the boundary of another open set $\widetilde{\Omega}$ ). In the counterexample provided here, $\partial \Omega$ is piecewise analytic and globally at most $\mathcal{C}^{1, \frac{1}{2}}$ as analyzed in 13 .

Proof of Theorem 2.1: Let $D=(-1,1)^{2}$ and $\alpha \in(\pi, 4)$. We will construct $\Omega$ as an optimal set for the shape minimization problem (3).

From [4. Theorem 2.4.6] (see also [10]), we know there exists a quasi-open optimal set $\Omega^{*}$ which solves problem (3). In view of [2, Corollary 1.2], $\Omega^{*}$ is in fact an open set. It is known that, for any open bounded set $\Omega$ (and in particular for $\Omega^{*}$ ), the functional $J$ defined in (4) satisfies

$$
J(\Omega)=\int_{\Omega}\left(\frac{1}{2}\left|\nabla u_{\Omega}\right|^{2}-u_{\Omega}\right) d x
$$

where $u_{\Omega}$ denotes the unique solution of the homogeneous Dirichlet problem

$$
\begin{cases}-\Delta u=1 & \text { in } \Omega \\ u=0 & \text { on } \partial \Omega .\end{cases}
$$

Since $\alpha<4, \Omega^{*}$ cannot be equal to $D$ so that the free boundary $\Gamma:=\partial \Omega^{*} \cap D$ is nonempty. Moreover, by [1, Section 5], we infer that $\Gamma$ is analytic because $f \equiv 1$ is positive and analytic. On this "free boundary" $\Gamma$, using the notion of shape derivative (see for instance [10]), we classically obtain the Euler-Lagrange equation of problem (3), namely, (6) with $\Omega=\Omega^{*}, u=u_{\Omega^{*}}$ together with

$$
\left|\nabla u_{\Omega^{*}}\right|=\Lambda>0 \text { on } \partial \Omega^{*} \cap D .
$$

Since $f(u)=1>0$, the positivity of the Lagrange multiplier $\Lambda$ follows from [1, Proposition 6.1]. By elliptic regularity, we know that there exists a unique solution $u_{\Omega^{*}} \in \mathcal{C}^{\infty}(\Omega \cup \Gamma)$ to (7).

We now prove the geometric properties of solutions of (3). First, since $\alpha>\pi$, $\Omega^{*}$ is not a disk. Second, we show that $\Omega^{*}$ is starshaped, or at least that it may be replaced by an optimal starshaped set. To this end, we introduce $\widetilde{\Omega}:=S_{X} S_{Y}\left(\Omega^{*}\right)$, where $S_{X}$ and $S_{Y}$ denote the Steiner symmetrization about the axes $O X$ and $O Y$ respectively, see e.g. 10, 12. Because of the symmetry of the square $D$ with respect to these axes, we have $\widetilde{\Omega} \subset D$. Moreover, $|\widetilde{\Omega}|=\left|\Omega^{*}\right|=\alpha$ and, by well-known properties of Steiner symmetrization, $J(\widetilde{\Omega}) \leq J\left(\Omega^{*}\right)$. Therefore, $\widetilde{\Omega}$ is also a solution of the shape optimization problem (3) so that, as for any optimal set, $\widetilde{\Gamma}=\partial \widetilde{\Omega} \cap D$ is smooth and $u_{\widetilde{\Omega}}$ satisfies (5). To verify that it is starshaped, we may denote

$$
\forall x \in[-1,1], \quad A(x):=\left\{y \in[-1,1] ;(x, y) \in S_{Y}\left(\Omega^{*}\right)\right\} .
$$

As a consequence of the definition of the Steiner symmetrization, we have $[0 \leq x \leq \hat{x}] \Rightarrow[A(\hat{x}) \subset$ $A(x)]$. We may also write

$$
S_{X} S_{Y}\left(\Omega^{*}\right)=\left\{(x, y) ;|y| \leq \frac{1}{2} \operatorname{meas} A(x)\right\}
$$

Since $x \in[0,1] \rightarrow$ meas $A(x)$ is nonincreasing, we have

$$
\left[|y| \leq \frac{1}{2} \operatorname{meas} A(x), \lambda \in(0,1)\right] \Rightarrow\left[|\lambda y| \leq \frac{1}{2} \operatorname{meas} A(x) \leq \frac{1}{2} \operatorname{meas} A(\lambda x)\right] .
$$

This proves that $\widetilde{\Omega}$ is starshaped.

Therefore, $\Omega=\widetilde{\Omega}, u=u_{\widetilde{\Omega}}, c=\Lambda, \Gamma=$ any connected component of $\partial \Omega \cap D$ satisfy the statement of Theorem 2.1.

We conclude this section by mentioning some possible extensions of Theorem 2.1.

Remark 2.4 The construction done in the proof of Theorem 2.1 is valid in any dimension and one finds as well an optimal open set $\Omega^{*} \subset(-1,1)^{n}$ (see [1] for a proof), which is different from a 
ball if $\alpha>\omega_{n}$ (the measure of the unit ball). But, the full regularity of the boundary is not proved -and probably does not hold- in any dimension. According to some recent papers ([5, 4, 15, 16]), it is very likely that full regularity of the boundary may be extended to dimensions greater than 2 (up to 6 ? but not more?).

However, as proved in [1], the reduced boundary of this $\Omega^{*}$ is an analytic hypersurface and this regular part of the boundary is of positive $(n-1)$-Hausdorff measure if $\alpha<2^{n}$, whereas $\Omega^{*}$ is not a ball if $\alpha>\omega_{n}$. Therefore, this also provides a (generalized) counterexample in any dimension by choosing $\Gamma$ to be this reduced boundary.

Remark 2.5 In view of [3] (see also [9, Section 3.4]), it is possible to extend the statement of Theorem 2.1 to the case when $J$ is replaced by the shape functional $\Omega \rightarrow \lambda_{1}(\Omega)$, the first eigenvalue of the Laplace operator on $\Omega$ with homogeneous Dirichlet boundary conditions. This provides one more example of an optimal domain $\Omega^{*}$ where $u_{\Omega^{*}}$, the first normalized eigenfunction, solves (1) with $f(u)=\lambda u$ (here, $\lambda=\lambda_{1}\left(\Omega^{*}\right)$ ). The proof is similar and we do not reproduce it here. It is possible that one could go further and extend the same construction to more general sources $f(u)$, for instance of power-type such as $f(u)=u^{p}$.

Remark 2.6 The minimal shape $\Omega^{*}$ for the second Dirichlet eigenvalue $\lambda_{2}(\Omega)$ of the Laplace operator, among all planar convex domains of given area, is also a natural candidate for another nice counterexample. It is proved that $\Omega^{*}$ is not a "stadium" (the convex envelope of two identical tangent balls), see [11. However, it is expected that it looks like a stadium (see 11]). If it is the case, as explained in [8], then the first order optimality condition would lead to an overdetermined problem in which the expected overdetermined part $\Gamma$ would be the strictly convex part of $\partial \Omega^{*}$. The exact regularity and shape of $\Omega^{*}$ is still to be completely understood: see [11, Theorems $\left.4,6,8\right]$ and 13$]$.

Remark 2.7 In the proof of Theorem 2.1, we started with some optimal shape $\Omega^{*}$ and adapted it so that it satisfies the required conditions. We may wonder whether all optimal shapes have the same symmetry properties. This question is related to the nontrivial question of equality case in the Steiner symmetrization, namely: is it true that $J(\Omega)=J\left(S_{X}(\Omega)\right)$ implies that $\Omega=S_{X}(\Omega)$ up to a translation? We refer to [6] for this question.

\section{Counterexamples via explicit construction}

In this section we provide an explicit example of a problem of type (2) which admits a solution on a domain different from a ball. We also exhibit a similar example for an analogous exterior problem.

\subsection{A counterexample in an interior domain}

Theorem 3.1 There exist a Lipschitz continuous and strictly increasing function $f: \mathbb{R} \rightarrow(0,+\infty)$ and $u \in \mathcal{C}^{2}(\bar{\Omega})$ solution of

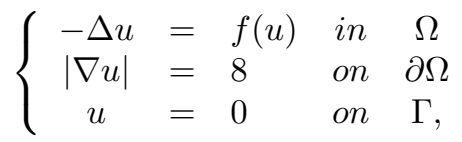

where $\Omega \subset \mathbb{R}^{n}$ is open, bounded, simply connected, different from a ball, with $\partial \Omega$ globally $\mathcal{C}^{\infty}$, and $\Gamma \subset \partial \Omega$ is nonempty, connected, relatively open and included in a sphere of $\mathbb{R}^{n}$.

Proof: Fix an integer $n \geq 2$ and consider the function $f: \mathbb{R} \rightarrow(0,+\infty)$ defined by

$$
f(s)= \begin{cases}\frac{64(n-1)}{8-s} & \text { if } s \leq 0 \\ 4[(n+2) \sqrt{s+4}-6] & \text { if } s \geq 0 .\end{cases}
$$




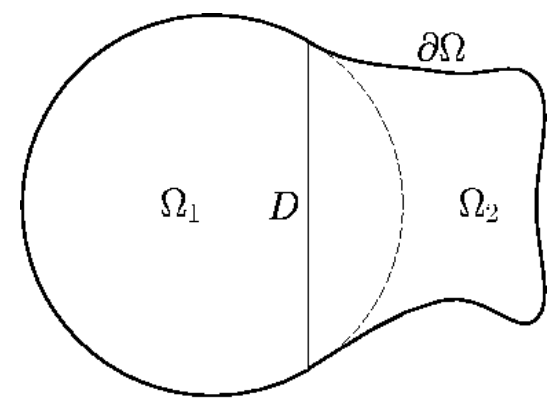

Figure 1 : domain $\Omega$ in Theorem 3.1.

Then, $f$ is globally Lipschitz continuous and strictly increasing over $\mathbb{R}$.

Consider also the (radial) function $u$ defined on $\mathbb{R}^{n}$ by

$$
u(x)= \begin{cases}\left(3-|x|^{2}\right)^{2}-4 & \text { if }|x| \leq 1 \\ 8(1-|x|) & \text { if }|x| \geq 1 .\end{cases}
$$

Then, $u \in \mathcal{C}^{2}\left(\mathbb{R}^{n}\right)$; to see this, it suffices to write $u=u(r)$ as a function of the real variable $r=|x|$ and to note that

$$
u^{\prime}(r)=\left\{\begin{array}{ll}
-4 r\left(3-r^{2}\right) & \text { if } r \leq 1 \\
-8 & \text { if } r \geq 1,
\end{array} \quad u^{\prime \prime}(r)= \begin{cases}-12+12 r^{2} & \text { if } r \leq 1 \\
0 & \text { if } r \geq 1\end{cases}\right.
$$

are continuous functions in $[0, \infty)$. Moreover, some computations show that $u$ satisfies

$$
-\Delta u=f(u) \quad \text { in } \mathbb{R}^{n}, \quad u=0 \quad \text { on } \partial B, \quad|\nabla u|=8 \quad \text { in } \mathbb{R}^{n} \backslash B,
$$

where $B$ denotes the unit ball.

Let $\Omega_{1}=\left\{x \in B ; x_{1}<\frac{1}{2}\right\}$ and $D=\left\{x \in B ; x_{1}=\frac{1}{2}\right\}$. Consider a bounded domain $\Omega_{2} \subset\{x \in$ $\left.\mathbb{R}^{n} ; x_{1}>\frac{1}{2}\right\}$ such that $D \subset \partial \Omega_{2}$ and $\left(\partial \Omega_{2} \backslash \bar{D}\right) \subset\left(\mathbb{R}^{n} \backslash \bar{B}\right)$. Let $\Omega=\Omega_{1} \cup D \cup \Omega_{2}$ (see Figure 1); for a suitable choice of $\Omega_{2}$ one has $\partial \Omega \in \mathcal{C}^{\infty}$. Let $\Gamma=\partial \Omega_{1} \cap \partial \Omega$, then $u$ satisfies (8) but $\Omega$ is not a ball.

Theorem 3.1 should be compared with the following result obtained in [8], and similar to Proposition 2.3:

Proposition 3.2 Let $\Omega$ be open and bounded with $\partial \Omega$ connected. Let $\Gamma \subset \partial \Omega$ nonempty and (relatively) open. Assume there exists an open set $\widetilde{\Omega}$ with a connected analytic boundary containing $\Gamma$. If there exists a solution $u$ of (8) with $f$ analytic and nonincreasing, then $\Omega=\widetilde{\Omega}, \Omega$ is a ball, and $u$ is radially symmetric.

Note in particular that: the overdetermined part $\Gamma$ in Theorem 3.1 satisfy the hypothesis in Proposition 3.2 (analytically continuable according to the definition in [8, Section 3.1]), but $f$ is neither analytic, nor nonincreasing.

Similarly, Theorem 3.1 should also be compared with the statements (b) in Theorems 3 and 7 in [8 which gives more various sufficient conditions to obtain symmetry in overdetermined problems of type (8). Again, Theorem 3.1 provides an example where all these hypothesis are satisfied, except the fact that $f$ be nonincreasing.

\subsection{A counterexample in an exterior domain}

Theorem 3.3 There exist a Lipschitz continuous function $f: \mathbb{R} \rightarrow \mathbb{R}$, and $u \in \mathcal{C}^{2}\left(\mathbb{R}^{n} \backslash \Omega\right)$ solution of

$$
\begin{cases}-\Delta u=f(u) & \text { in } \mathbb{R}^{n} \backslash \bar{\Omega} \\ |\nabla u|=\frac{1}{2} & \text { on } \partial \Omega \\ u=1 & \text { on } \Gamma \\ u \rightarrow 0,|\nabla u| \rightarrow 0 & \text { as }|x| \rightarrow \infty,\end{cases}
$$


where $\Omega \subset \mathbb{R}^{n}$ is open, bounded, simply connected, different from a ball, with $\partial \Omega$ globally $\mathcal{C}^{\infty}$, and $\Gamma \subset \partial \Omega$ is nonempty, connected, relatively open and included in a sphere.

Proof: Fix an integer $n \geq 2$ and consider the function $f: \mathbb{R} \rightarrow \mathbb{R}$ defined by

$$
f(s)= \begin{cases}\frac{n-1}{2(3-2 s)} & \text { if } 1 \leq s<\frac{3}{2} \\ \frac{3(n-3)}{16}(3-\sqrt{9-8 s})^{3}-\frac{n-4}{16}(3-\sqrt{9-8 s})^{4} & \text { if } 0<s \leq 1 .\end{cases}
$$

Then, $f$ is globally Lipschitz continuous over $\left(0, \frac{3}{2}\right)$; moreover, if $n \geq 4$ then $f$ is positive and strictly increasing.

Consider also the (radial) function $u$ defined on $\mathbb{R}^{n} \backslash\{0\}$ by

$$
u(x)= \begin{cases}\frac{3-|x|}{2} & \text { if }|x| \leq 1 \\ \frac{3}{2|x|}-\frac{1}{2|x|^{2}} & \text { if }|x| \geq 1 .\end{cases}
$$

Then, $u \in \mathcal{C}^{2}\left(\mathbb{R}^{n} \backslash\{0\}\right)$; to see this, it suffices to write $u=u(r)$ as a function of the real variable $r=|x|$ and to note that

$$
u^{\prime}(r)=\left\{\begin{array}{ll}
-\frac{1}{2} & \text { if } 0<r \leq 1 \\
-\frac{3}{2 r^{2}}+\frac{1}{r^{3}} & \text { if } r \geq 1,
\end{array} \quad u^{\prime \prime}(r)= \begin{cases}0 & \text { if } 0<r \leq 1 \\
\frac{3}{r^{3}}-\frac{3}{r^{4}} & \text { if } r \geq 1\end{cases}\right.
$$

are continuous functions in $(0, \infty)$. Moreover, some computations show that $u$ satisfies

$$
-\Delta u=f(u) \quad \text { in } \mathbb{R}^{n} \backslash\{0\}, \quad u=1 \quad \text { on } \partial B, \quad|\nabla u|=\frac{1}{2} \quad \text { in } \bar{B} \backslash\{0\},
$$

where $B$ denotes the unit ball. Take any smooth domain $\Omega \subsetneq B$ such that $0 \in \Omega$ and $\left\{x \in \partial B ; x_{1}<\right.$ $\left.\frac{1}{2}\right\} \subset \partial \Omega$ (see figure 2). Let $\Gamma=\partial \Omega \cap \partial B$, then $u$ satisfies (9) but $\Omega$ is not a ball.

Remark 3.4 Again, Theorem 3.3 should be compared with the results in [8], similarly to what we did for Theorem 3.1.

\section{References}

[1] T. Briançon, Regularity of optimal shapes for the Dirichlet's energy with volume constraint, ESAIM: Control, Optimization and Calculus of Variations 10, 2004, 99-122

[2] T. Briançon, M. Hayouni and M. Pierre, Lipschitz continuity of state functions in some optimal shaping, Calc. Var. PDE 23, 2005, 13-32

[3] T. Briançon and J. Lamboley, Regularity of the optimal shape for the first eigenvalue of the Laplacian with volume and inclusion constraint, to appear in Annales de l'Institut Henri Poincaré. Analyse Non Linéaire, 2008

[4] G. Buttazzo and G. Dal Maso, An existence result for a class of shape optimization problems, Arch. Rat. Mech. Anal. 122, 1993, 183-195

[5] L.A. Caffarelli, D. Jerison and C.E. Kenig, Global energy minimizers for free boundary problems and full regularity in three dimensions, Contemp. Math. 350, Amer. Math. Soc., Providence, RI (2004), 83-97

[6] A. Cianchi, N. Fusco, Steiner symmetric extremals problems in Pólya-Szegö-type inequalities, Advances in Math. 203, 2006, 673-728 


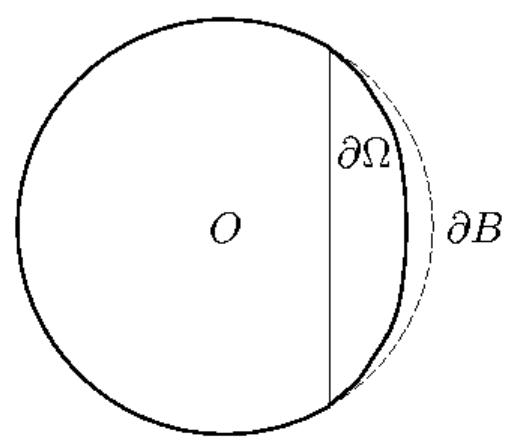

Figure $2:$ domain $\Omega$ in Theorem 3.3 .

[7] D. De Silva and D. Jerison, A singular energy minimizing free boundary, to appear in J. Reine Angew. Math., 2008

[8] I. Fragalà and F. Gazzola, Partially overdetermined problems, to appear in J. Diff. Eq.

[9] A. Henrot, Extremum problems for eigenvalues of elliptic operators, Frontiers in Mathematics, Birkhauser 2006

[10] A. Henrot and M. Pierre, Variation et optimisation de forme, Springer 2005

[11] A. Henrot and E. Oudet, Minimizing the second eigenvalue of the Laplace operator with Dirichlet boundary conditions, Arch. Rat. Mech. Anal. 169, 2003, 73-87

[12] B. Kawohl, Rearrangements and convexity of level sets in p.d.e., Springer Lecture Notes in Maths 1150, 1985 
[13] J. Lamboley, About Hölder regularity of the optimal convex planar shape for $\lambda_{2}$, Preprint, 2008

[14] J. Serrin, A symmetry problem in potential theory, Arch. Ration. Mech. Anal. 43, 1971, 304-318

[15] G.S. Weiss, Partial regularity for weak solutions of an elliptic free boundary problem, Comm. Part. Diff. Eq. 23, 1998, 439-457

[16] G.S. Weiss, Partial regularity for a minimum problem with free boundary, Journal Geom. Anal. 9, 1999, 317-326

Ilaria Fragalà

Dipartimento di Matematica

Politecnico di Milano

Piazza Leonardo da Vinci 32,

20133 Milano, Italy

ilaria.fragala@polimi.it

Jimmy Lamboley

ENS Cachan Bretagne

IRMAR, UEB,

Campus de Ker Lann,

35170 Bruz, France

jimmy.lamboley@bretagne.ens-cachan.fr
Filippo Gazzola

Dipartimento di Matematica

Politecnico di Milano

Piazza Leonardo da Vinci 32,

20133 Milano, Italy

filippo.gazzola@polimi.it

Michel Pierre

ENS Cachan Bretagne

IRMAR, UEB,

Campus de Ker Lann,

35170 Bruz, France

michel.pierre@bretagne.ens-cachan.fr 